\title{
Expression of gastric pyloric mucin, MUC6, in colorectal serrated polyps
}

Angela N Bartley ${ }^{1,2}$, Patricia A Thompson ${ }^{3}$, Julie A Buckmeier ${ }^{3}$, Carole Y Kepler ${ }^{3}$, Chiu-Hsieh $\mathrm{Hsu}^{3}$, Manuel S Snyder ${ }^{3}$, Peter Lance ${ }^{3}$, Achyut Bhattacharyya ${ }^{1}$ and Stanley R Hamilton ${ }^{2}$

${ }^{1}$ Department of Pathology, University of Arizona, Tucson, AZ, USA; ${ }^{2}$ Department of Pathology, Division of Pathology and Laboratory Medicine, The University of Texas MD Anderson Cancer Center, Houston, TX, USA and ${ }^{3}$ Arizona Cancer Center, Mel and Enid Zuckerman College of Public Health, University of Arizona,

Tucson, AZ, USA

Serrated polyps of the colorectal mucosa represent a heterogeneous and controversial taxonomic category with variation in histopathological, molecular, and immunohistochemical characteristics and with an incomplete understanding of pathogenesis. A previous study reported that the expression of gastric pylorictype mucin, MUC6, characterized sessile serrated adenomas. We therefore evaluated the expression of MUC6 in serrated polyps identified among 2502 participants in a Phase III chemoprevention trial within the Arizona Cancer Center Colorectal Cancer Prevention Trials Program and characterized the associated histopathological features and location. We carried out immunohistochemistry for MUC6 on 146 serrated lesions and 87 conventional tubular adenomas, and assessed the percentage of cells with expression and the grade of staining intensity. In all 92 hyperplastic polyps, 43 sessile serrated adenomas, and 11 traditional serrated adenomas were included. Polyps ranged in size from 1-150 mm. The association of MUC6 staining with serrated polyp category was evaluated using classification and regression tree (CART) analysis and two-sided Fisher's exact test. A total of $53 \%$ of sessile serrated adenomas $(n=23), 17 \%$ of hyperplastic polyps $(n=16)$, and $18 \%$ of traditional serrated adenomas $(n=2)$, but none of 87 tubular adenomas, expressed MUC6. Expression was limited to the lower crypts in all serrated polyps. The extent of positive staining ranged from $2-100 \%$ of crypt cells and was independent of the histopathological type. MUC6 expression had relatively high specificity for sessile serrated adenoma (82\%) but low sensitivity (54\%). In CART analysis, proximal location was found to be the best partitioning factor for MUC6, followed by classification as sessile serrated adenoma. We conclude that MUC6 expression is strongly associated with proximal location of serrated polyps, but only has modest utility as a tissue biomarker for sessile serrated adenoma.

Modern Pathology (2010) 23, 169-176; doi:10.1038/modpathol.2009.155; published online 23 October 2009

Keywords: serrated adenoma; hyperplastic polyp; colonic neoplasms; MUC6

Serrated adenomas of the colorectum were first described in 1990 by Longacre and FenoglioPreiser. ${ }^{1}$ Since the initial description, many attempts have been made to distinguish serrated lesions at the histopathological, immunological, and molecular levels. ${ }^{2-15}$ Although there has been great inter-observer variation in classifying serrated

Correspondence: Dr AN Bartley, Department of Pathology, The University of Texas M.D. Anderson Cancer Center, Division of Pathology and Laboratory Medicine, 1515 Holcombe Blvd, Unit 085, Houston, TX, 77030, USA.

E-mail: anbartley@mdanderson.org

Received 16 June 2009; revised 9 September 2009; accepted 15 September 2009; published online 23 October 2009 lesions, distinguishing between these and other polyps of the colorectum has become increasingly important. Of particular, clinical relevance is the proposed origin of sporadic colorectal cancers with high levels of microsatellite instability (MSI-H) from hyperplastic polyps and sessile serrated adenomas. The recent suggestion that hyperplastic polyps of the proximal colon can serve as the immediate precursor for this molecular subtype of colonic carcinoma raised questions about the inherent malignant potential of hyperplastic polyps previously thought to be benign lesions. ${ }^{16}$

Recently, the aberrant expression of mucin (MUC) gene products in adenomas and carcinomas of the 
colorectum has been investigated as a potential source of tissue biomarkers to improve serrated polyp subclassification. ${ }^{5,6}$ Mucins are high molecular-weight epithelial glycoproteins that are classified into two distinct structural and functional classes: secreted gel-forming mucins and transmembrane mucins. Twenty MUC genes have been identified or sequenced to date. The secreted gel-forming mucins include products of MUC2, MUC5AC, $M U C 5 B$, and MUC6 genes that are clustered on chromosome 11p15.5. These mucins have a role in the normal physiological processes of the gastrointestinal tract, and in neoplastic progression and metastasis of colon cancer cells. ${ }^{17}$ MUC2 and MUC5AC are expressed in normal colonic epithelium and in colorectal cancers, whereas MUC5B is only expressed to a minor degree in the colon. ${ }^{17}$ MUC6 has little expression in the colon, but is expressed in surface foveolar epithelium and deep antral/pyloric glands of the stomach. ${ }^{18}$

Phenotypically, the cells lining the crypts of hyperplastic polyps and serrated adenomas show differentiation similar to gastric pyloric gland mucous cells. Recent studies have examined the differential expression of MUC gene products, including MUC6, to distinguish sessile serrated adenomas from hyperplastic polyps to improve classification. ${ }^{19-23}$ Owens et $a l^{23}$, for example, recently reported $100 \%$ specificity of MUC6 to discriminate between sessile serrated adenoma and hyperplastic polyp. In order to provide additional data on the clinical utility of MUC6 as an adjunct tissue biomarker, we examined its expression in relation to histopathological classification, size, and anatomic location of colorectal serrated lesions in our large studies of subjects with colorectal adenomas, who were enrolled in Phase III chemoprevention trials.

\section{Materials and methods}

\section{Patients and Specimens}

We examined serrated polyps pooled from 2502 subjects participating in the Wheat Bran Fiber and the Ursodeoxycholic Acid Phase III polyp prevention trials conducted at the Arizona Cancer Center, the details of which have been reported elsewhere. ${ }^{24,25}$ Briefly, eligibility criteria included the removal of one or more colorectal adenomas during a colonoscopic examination within the 6-month period before study registration. All colon neoplasms must have been removed completely, except for diminutive $(<3 \mathrm{~mm})$ sessile rectal polyps. Subjects with a familial colorectal cancer syndrome or evidence of hyperplastic polyposis were excluded from the trials. Baseline characteristics of the patients enrolled in the two studies are presented in Table 1.

The serrated polyps were identified through review of the biopsy or polypectomy specimens of 3901 baseline and recurrent lesions. Eighty-seven
Table 1 Baseline characteristics of all patients participating in wheat bran fiber (WBF) and the ursodeoxycholic acid (UDCA) phase III polyp prevention trials

\begin{tabular}{lcc}
\hline & $\begin{array}{c}W B F \\
(\mathrm{~N}=1304)\end{array}$ & $\begin{array}{c}\text { UDCA } \\
(\mathrm{N}=1193)\end{array}$ \\
\hline Age, mean (s.d.) (years) & $65.7 \pm 8.8$ & $65.8 \pm 8.5$ \\
& & \\
Sex (\%) & 66.8 & 67.5 \\
Male & 33.2 & 32.5 \\
Female & $1.5 \pm 0.9$ & $1.7 \pm 1.1$ \\
Mean no. of adenomas & & \\
& & \\
Location (\%) & 46.8 & 37.2 \\
Distal colorectum & 31.8 & 36.7 \\
Proximal only & 15.3 & 19.7 \\
Proximal and distal & 6.1 & 6.4 \\
Unknown & $8.3 \pm 5.9$ & $8.9 \pm 5.9$ \\
Size of largest adenoma, mean & & \\
(s.d.) (mm) & & \\
& & \\
Adenoma histology (\%) & 78.7 & 20.3 \\
$\quad$ Tubular & 21.3 & \\
Tubulovillous/villous & & \\
\hline
\end{tabular}

conventional tubular adenomas with low-grade dysplasia were also selected for comparison from the same study population. None of the tubular adenomas contained high-grade dysplasia. All other histopathological types of polyps were excluded including inflammatory, hamartomatous, and juvenile polyps.

Only those serrated polyps with at least a portion of well-oriented, full-thickness serrated mucosa in the histopathological sections were included. Most of the specimens contained adjacent non-lesional colorectal mucosa. Samples with cautery artifact involving the entire serrated epithelial component due to colonoscopic polypectomy or 'hot' biopsy procedure were excluded from the study. These inclusion and exclusion criteria resulted in a sample size of 161 serrated polyps. Age and gender of patient, as well as size of the lesion and location were recorded. Size was obtained from the colonoscopic measurement or by measurement of the lesion on the histopathological section if the size was greater than what was reported. Polyp site was categorized as distal or proximal relative to the splenic flexure. We were unable to confirm definitively from the endoscopy report the location of 15 serrated polyps, so these were excluded from further analyses. A total of 146 serrated polyps remained for MUC6 staining.

The study was approved by the Institutional Review Boards of the University of Arizona and The University of Texas M. D. Anderson Cancer Center.

\section{Histopathological Criteria}

The classification of sessile serrated adenoma was based on the following criteria of Snover et $a l^{12}$ : branching of crypts, dilatation of the base of the 
crypts, crypts extending parallel to the muscularis mucosae, presence of mature cells with gastric foveolar cell phenotype at the base replacing the proliferative zone of non-lesional mucosa, serration at the base of the crypts, and, less commonly, pseudostratification and eosinophilic change of the surface epithelium. Hyperplastic polyps were classified on the basis of serration in only the upper half to one-third of the crypts with normal proliferation including a proliferative zone at the base of the crypts that was symmetric and continuous, and the crypts remaining narrow and lined with proliferative cells. ${ }^{8}$ Traditional serrated adenomas were classified on the basis of villiform configuration that appeared protuberant rather than sessile with a uniform population of abnormal columnar epithelium containing eosinophilic cytoplasm, centrally placed elongated nuclei that were somewhat hyperchromatic, and mild pseudostratification. ${ }^{12}$ All histopathological evaluations were carried out by two gastrointestinal pathology pathologists (AB and SRH) without the knowledge of any clinical characteristics.

\section{Immunohistochemical Analysis of MUC6}

Archival paraffin-embedded tissue blocks from each case when available $(n=111$ serrated polyps, $n=60$ tubular adenomas) or tissue microarray blocks constructed for cases, for which return of blocks was mandated by the enrolling institution $(n=35$ serrated polyps, $n=27$ tubular adenomas) were used for immunohistochemistry. The tissue microarrays were constructed using a Beecher instrument from histopathological areas selected in scout $\mathrm{H} \& \mathrm{E}$ slides from the specimen blocks that were used for classification. The cores were $1.5 \mathrm{~mm}$ in diameter, and the tissue microarray blocks included gastric and colorectal mucosa as positive and negative controls along with sessile serrated adenoma, hyperplastic polyp, and tubular adenomas with low-grade intra-epithelial neoplasia (dysplasia).

Sections were cut at $5-\mu \mathrm{m}$ thickness onto positively charged slides and stained with MUC6 mouse monoclonal antibody VP-M658 (Vector Laboratories, Burlingame, CA, USA) at a dilution of 1:40. The immunogen for the antibody was a synthetic peptide of the MUC6 tandem repeat sequence purified by high-performance liquid chromatography. Gastric antral mucosa was used as a positive control with the cytoplasmic staining pattern in the glands. Negative controls consisted of adjoining TMA sections with primary antibodies that were classmatched mouse IgG proteins. Deparaffinization with xylene, antigen retrieval with ethylene diamine tetraacetic acid (EDTA), and immunohistochemistry were carried out using the Discovery XT Automated immunohistochemistry system (Ventana Medical Systems, Tucson, AZ, USA). Primary antibody staining, detection and amplification with 3,3- o-diamnobenzidine (DAB) chromogen, and hematoxylin counterstaining were carried out using a multimer based detection system (UltraMap, Ventana Medical Systems, Tucson, Arizona, USA), with validated reagents. After staining on the instrument, slides were dehydrated through graded alcohols to xylene and a coverslip applied with Pro-Texx mounting medium (Thermo Scientific, Waltham, MA, USA).

Immunoreactivity for MUC6 was evaluated as the percentage of cells with expression and the grade of staining intensity. The extent of staining was assessed as an estimated percentage of total positively stained areas within the lesion, and grade of intensity as weak, moderate, or strong for each positively stained lesion. Predominant expression patterns for MUC6 staining were defined as apical, basolateral, supranuclear, or diffuse.

\section{Statistical Analysis}

The clinicopathological features were summarized by calculating mean, median, s.d., and range for continuous features (eg, polyp size), as well as calculating frequencies and relative percentages for categorical features (eg, histopathological type). MUC6 staining was first summarized by calculating mean, median, s.d., and range, ie minimum and maximum, of estimated staining percentage. A twosided Fisher's exact test was carried out to evaluate the statistical significance of differences for categorical outcomes (eg, large lesion defined as $\geqslant 1 \mathrm{~cm}$ ), and a Wilcoxon's rank-sum test for continuous outcomes (eg, lesion size). All significance levels were set at $5 \%$. In addition, sensitivity and specificity of MUC6 staining for sessile serrated adenoma were calculated.

A classification and regression tree (CART) analysis, wherein a deviance method was used to measure the impurity of node, was then carried out to explore the factors and the possible interactions between the factors associated with positive MUC6 staining. A multivariate logistic regression model was also fitted to the status of MUC6 staining to compare with the findings from the CART analysis and to study the significance of the factors associated with positive MUC6 staining.

\section{Results}

The clinicopathological features of the colorectal serrated polyps in our study are presented in Table 2. Of the 146 serrated polyps, 43 were classified as sessile serrated adenoma (17 proximal, 26 distal), 92 as hyperplastic polyp (20 proximal, 72 distal), and 11 as traditional serrated adenoma (4 proximal, 7 distal). These polyps originated from 24 women and 82 men with a mean age of 64 years (range 45-78 years). No significant associations were seen with gender or age. Polyp size ranged from $1-150 \mathrm{~mm}$. There were statistically significant dif- 
Table 2 Clinical characteristics of patients with serrated polyps

\begin{tabular}{lccccc}
\hline Histology type & \# Polyps & \# Patients, mean (range) & Age (mean \pm s.d.) & Male (\%) & Proximal (\%) \\
\hline $\begin{array}{l}\text { Hyperplastic polyp } \\
\text { Sessile serrated adenoma }\end{array}$ & 92 & $68,1.3(1-5)$ & $63.4(7.9)$ & 80.9 & 19.6 \\
Traditional serrated & 43 & $35,1.3(1-5)$ & $66.3(7.8)$ & 72.4 & 44.2 \\
$\begin{array}{l}\text { Adenoma } \\
\text { All serrated }\end{array}$ & 11 & $9,1.2(1-3)$ & $66.7(6.2)$ & 66.7 & 36.4 \\
& 146 & *106, 1.4 (1-5) & $64.5(7.8)$ & 77.4 \\
\hline
\end{tabular}

The number of total polyps differs from the number of patients, as some patients contributed more than one polyp to the sample set.

Table 3 Relation between histopathologic polyp type, number of polyps with positive mucin (MUC6) expression, and percentage of crypt cells positive for MUC6

\begin{tabular}{lccc}
\hline & \multicolumn{2}{c}{ Histology } \\
\cline { 2 - 4 } & Hyperplastic polyp & $\begin{array}{c}\text { Sessile serrated } \\
\text { adenoma }\end{array}$ & $\begin{array}{c}\text { Traditional serrated } \\
\text { adenoma }\end{array}$ \\
\hline MUC6 $^{+} ; n(\%)$ & $16 / 92(17.4 \%)$ & $23 / 43(53.5 \%)$ & $2 / 11(18.2 \%)$ \\
Crypts staining MUC6 $^{+}$Mean (range)* & $26.4 \%(2-90 \%)$ & $26.8 \%(2-90 \%)$ & $52.5 \%(5-100 \%)$ \\
\hline
\end{tabular}

ferences in lesion size between sessile serrated adenomas (median $5 \mathrm{~mm}$ ) and hyperplastic polyps (median $4 \mathrm{~mm}, P=0.0002$ from Wilcoxon's ranksum test). However, differences in the frequency of clinically relevant large lesions of $1 \mathrm{~cm}$ or more between sessile serrated adenoma $(11.6 \%)$ and hyperplastic polyp $(6.5 \%)$ did not reach statistical significance $(P=0.33$ by Fisher's exact test). No intra-epithelial neoplasia (dysplasia) was seen in any sessile serrated adenoma or hyperplastic polyp, and all tubular adenomas selected for comparison had only low-grade dysplasia.

In the sample set of 146 polyps, all lesions that were positive for MUC6 showed only basolateral and diffuse staining patterns (Table 3). 23 of 43 sessile serrated adenomas (53\%), 16 of 92 hyperplastic polyps $(17 \%)$, and 2 of 11 traditional serrated adenomas (18\%) had basal staining for MUC6 (Figure 1a-d). None of the tubular adenomas expressed MUC6. Basal MUC6 staining exhibited high specificity for SSA (82\%), but low sensitivity $(54 \%)$.

Of the lesions staining positive, the percentage of crypt cells expressing MUC6 ranged from 2-100\%, and all positive lesions showed moderate to strong staining intensity. No significant differences were seen in staining intensity of the MUC6-expressing polyps in relation to their size, anatomic site, or histopathological subtype. Non-lesional colorectal mucosa, either adjacent to serrated lesions or as a separate specimen, was uniformly negative for MUC6.

A CART analysis was carried out to explore the factors associated with MUC6 expression status without relying on a specific distribution or model. Lesion characteristics (eg, anatomic location as proximal or distal, and lesion morphology as hyperplastic polyp, sessile serrated adenoma, or traditional serrated adenoma) and the correspond- ing demographic information for each patient (eg, age and gender) were the independent variables (predictors) to build the tree for MUC6 staining status. The results are shown in Figure 2. The analysis indicates that anatomic location (proximal vs distal) was the optimal first split, followed by lesion morphology, where traditional serrated adenoma and hyperplastic polyp were grouped together by the splitting algorithm (ie, sessile serrated adenoma vs hyperplastic polyp/traditional serrated adenoma).

Based on a multivariate logistic regression model (Table 4), proximal lesions had a significantly higher rate of MUC6 expression compared with distal lesions with an odds ratio of $5.79(P=0.0001$; 95\% CI 2.35-14.25), and sessile serrated adenoma had a significantly higher rate of MUC6 expression compared with hyperplastic polyp with an odds ratio of $5.18(P=0.001 ; 95 \%$ CI $2.04-13.15)$. MUC6 positivity did occur in distal sessile serrated adenomas ( 7 of $26,27 \%$ ) and hyperplastic polyps (11 of $72,15 \%$ ), but to a lesser extent than proximal lesions and with no significant association for sessile serrated adenoma compared with hyperplastic polyp or traditional serrated adenoma. To account for potential dependence between polyps within the same subject, analyses were conducted using a generalized linear mixed effects model with random intercept. These results were nearly identical to those shown for the analysis assuming independence between polyps within the same subject in Table 4.

\section{Discussion}

We demonstrate that gastric pyloric mucin MUC6 is expressed in all the categories of serrated lesions 


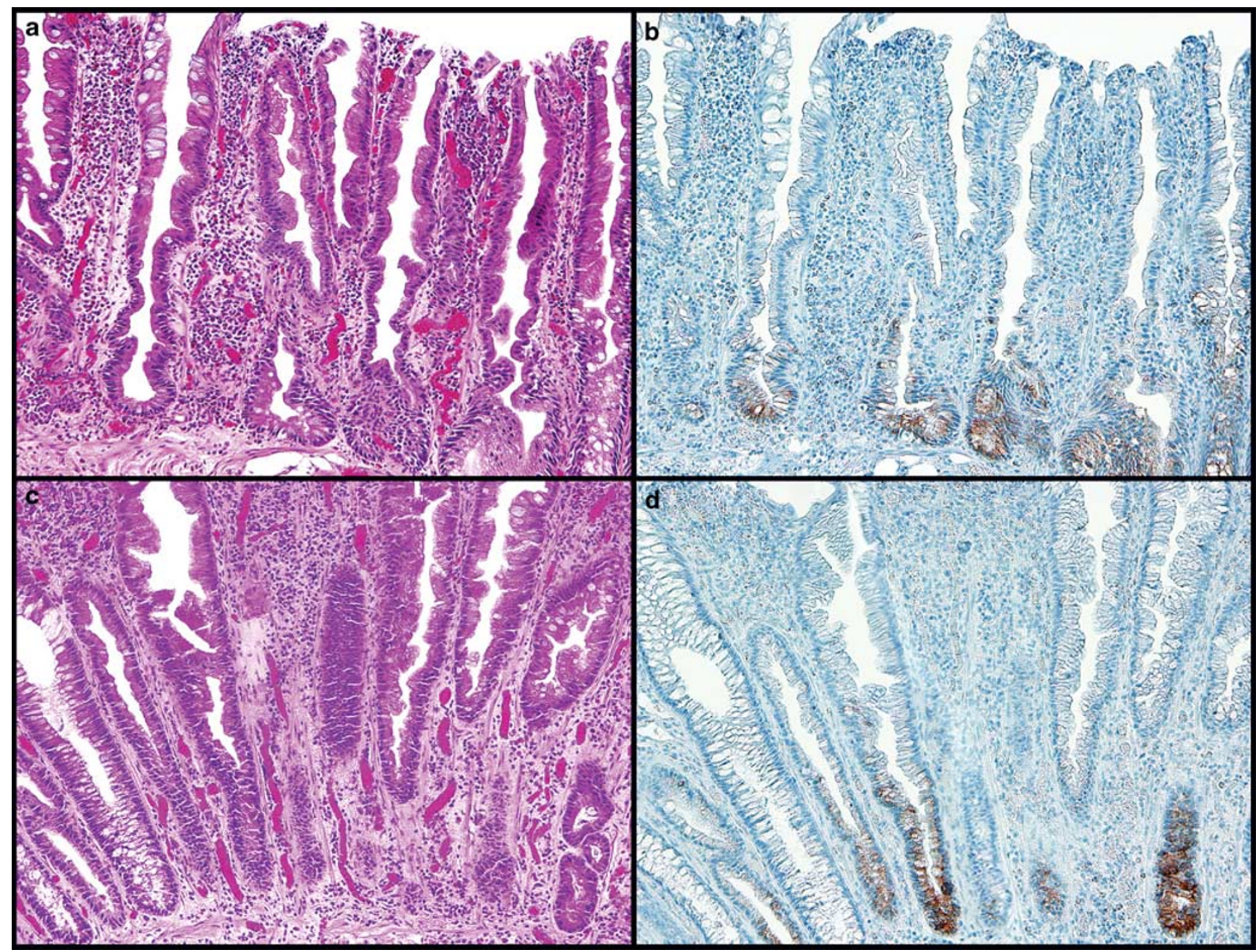

Figure 1 (a and b) Sessile serrated adenoma, hematoxylin and eosin (H\&E), proximal colon, $\times 20$. (a) Section of proximal colon stained with H\&E showing some features of sessile serrated adenoma including dilatation of the base of the crypts, crypts extending parallel to the muscularis mucosae, serration at the base of the crypts, and eosinophilic change of the surface epithelium. (b) Section of proximal colon sessile serrated adenoma in panel a with mucin (MUC6) immunostaining of the basal crypts. (c and d) Hyperplastic polyp, H\&E, distal colon, $\times 20$. (c) Section of distal colon stained with H\&E showing features of hyperplastic polyp including serration in the upper half to one-third of the crypts with normal proliferation including a proliferation zone at the base of the crypts that is symmetric, and crypts that remain narrow and lined with proliferative cells. (d) Section of distal colon hyperplastic polyp in panel c with MUC6 immunostaining of the basal crypts.

of the colorectum, including sessile serrated adenoma, hyperplastic polyp, and traditional serrated adenoma, but not in conventional tubular adenomas in our study. The basal expression of MUC6 was shown by immunohistochemistry to be more frequent in proximal serrated lesions regardless of category. Expression was more often positive in sessile serrated adenomas of the proximal colon with high specificity (82\%). However, basal MUC6 expression was seen in proximal hyperplastic polyps and in distal serrated polyps including both sessile serrated adenomas and hyperplastic polyps. MUC6 staining was not seen in distal traditional serrated adenomas. Patient gender, age, or size of the polyp was not significantly associated with MUC6 expression.

Our results are in contrast with those of Owens et $a l^{23}$ who reported $100 \%$ specificity when using
MUC6 to distinguish sessile serrated adenomas from hyperplastic polyps. In their study, none of the 48 hyperplastic polyps or 13 traditional serrated adenomas were positive for MUC6. Neither anatomic location nor polyp size appeared to account for the apparent differences in MUC6 expression. Also in their study, the authors found sessile serrated adenomas to be significantly larger than hyperplastic polyps and theorized that MUC6 staining could be related to polyp size rather than morphological polyp subtype. Although they found that sessile serrated adenomas tended to be slightly larger than the hyperplastic polyps, ${ }^{23}$ we did not find the difference to be clinically meaningful.

Percinel et $a l^{22}$ similarly found no MUC6 expression in hyperplastic polyps or normal mucosa. Twenty percent of the sessile serrated adenomas in their study were positive for MUC6. These authors 
Classification and Regression Tree (CART) for Basal MUC6 Expression
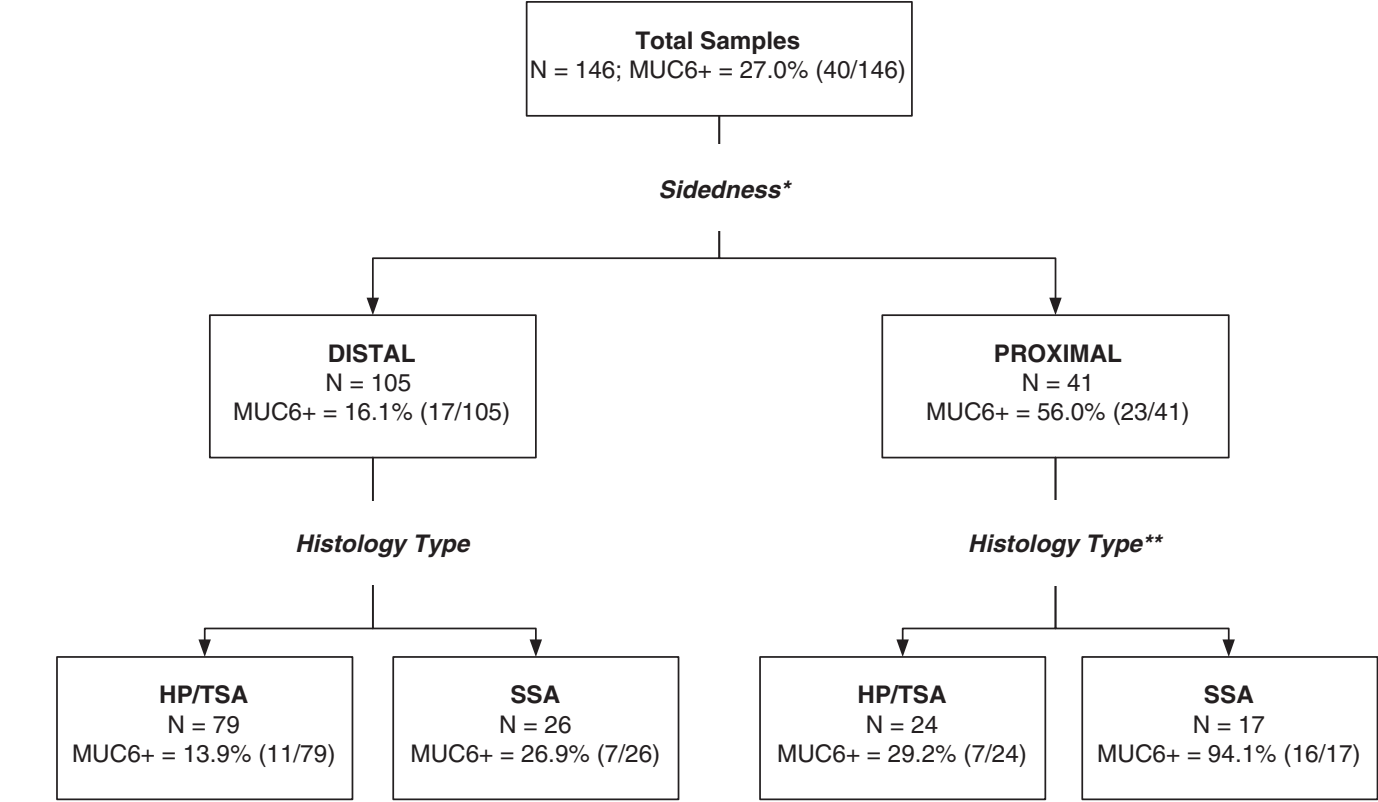

$H P(N=72 ; M U C 6+=15.2 \%(11 / 72))$

$H P(N=20 ; M U C 6+=25 \%(5 / 20))$

TSA $(N=7 ;$ MUC6+ $=0 \%(0 / 7))$

TSA $(N=4 ;$ MUC6+ $=50 \%(2 / 4))$

Figure 2 Classification and regression tree (CART) model of basal mucin (MUC6) staining and patient clinical characteristics. The model indicates that a polyp staining positive for MUC6 was explained by an interaction between proximal location and sessile serrated adenoma category. When sidedness was proximal, the proportion of MUC6-positive specimens was $56.0 \%$ compared with $16.1 \%$ in specimens from the distal colon. When histology was included, 94.1\% of specimens that were MUC6-positive in the proximal colon were sessile serrated adenomas compared with only $29.2 \%$ of proximal specimens that were hyperplastic polyps or traditional serrated adenomas. In the distal specimens, the corresponding frequencies were 26.9 and $13.9 \%$ No further splits were found to significantly improve the homogeneity of the subgroup outcomes for MUC6 staining. HP-hyperplastic polyp, SSA-sessile serrated adenoma, and TSAtraditional serrated adenoma.

Table 4 Association of clinicopathological features and mucin (MUC6) expression

\begin{tabular}{lll}
\hline Variable & Odds ratio $(95 \%$ CI) & P-value \\
\hline Age ( $\geq 64$ years) & $0.34(0.14-0.86)$ & 0.02 \\
Male vs female & $0.52(0.19-1.46)$ & 0.21 \\
Proximal vs distal & $5.79(2.35-14.25)$ & 0.0001 \\
& & \\
Histology Type & $1.06(0.17-6.53)$ & 0.95 \\
TSA vs HP & $5.18(2.04-13.15)$ & 0.001 \\
SSA vs HP & $0.21(0.03-1.26)$ & 0.09 \\
TSA vs SSA & \\
\hline
\end{tabular}

HP, hyperplastic polyp; SSA, sessile serrated adenoma; TSA, traditional serrated adenoma.

Age ( $\geq 64$ years), gender, location, and histology type were included in the multivariate logistic regression model.

concluded that hyperplastic epithelium may give rise to adenomatous change, and that MUC6 may later take part in the neoplastic continuum. Bartman et $a l^{19}$ found no MUC6 expression in hyperplastic polyps or normal mucosa by immunohistochemistry and found minimal amounts of MUC6 mRNA in samples of normal mucosa by slot-blot analysis. They found significantly higher levels of MUC6 mRNA in adenomas, but did not specifically analyze sessile serrated adenomas or hyperplastic polyps.
In contrast to these reports, we found MUC6 positivity in $17 \%$ of hyperplastic polyps regardless of colorectal location. This finding suggests that MUC6 lacks sufficient discrimination for use as a diagnostic tool to definitively differentiate sessile serrated adenoma from hyperplastic polyp. In agreement with our findings, Hirono et $a l^{20}$ found MUC6 expression in 68\% of hyperplastic polyps, $71 \%$ of sessile serrated adenomas, and $9.5 \%$ of tubular adenomas, and Mochizuka et $a l^{21}$ found MUC6 expression in $27 \%$ of hyperplastic polyps and $76 \%$ of sessile serrated adenomas. In their study, sessile serrated adenomas had significantly higher staining scores for MUC6. In addition, rightsided sessile serrated adenomas had a higher staining score than left-sided sessile serrated adenomas, but there was no significant difference between right-sided and left-sided hyperplastic polyps. Neither study found MUC6 expression in non-lesional colonic mucosa.

Also in agreement with several previous studies, ${ }^{2,8-10,21,22}$ we found that sessile serrated adenomas occurred at higher frequency in the distal colon than in the proximal colon, but sessile serrated adenomas showed a greater predilection for the proximal colon when compared with hyperplastic polyps. Other studies show sessile 
serrated adenomas predominantly located in the proximal colon. ${ }^{23,26}$ These differences could be attributed to sample selection as well as the use of differing diagnostic criteria for classifying serrated polyps. Nonetheless, the detection of MUC6 in distal and proximal sessile serrated adenomas, as well as hyperplastic polyps, limits the utility of MUC6 immunostaining to differentiate between these morphological subtypes.

Potential limitations of our study that may explain differences from other studies include the selection of a patient population that includes only those patients with known previous adenomas and the age of the histological blocks used in the study (ranging from 1991-2007). Methodological differences in the immunohistochemical preparation of the slides, including the antibodies used and staining methods that are not uniform in all of the studies, could contribute as well. These limitations, however, cannot explain the strong association with anatomic location in the proximal colon nor the clearly positive staining of serrated polyps other than sessile serrated adenomas. The low sensitivity in our sample, however, most likely reflects the older age of our specimens and failure to retrieve antigen, effects that are random and not likely to contribute to significant bias in the overall study results, with the exception of the sensitivity of the antibody method of MUC6 staining.

In summary, this is one of the largest study groups to date on MUC6 immunostaining in serrated polyps of the colorectum. Although we confirm some of the findings of smaller studies in the recent literature, including the predominant association of MUC6 with proximal or right-sided lesions, we did not observe exclusivity of MUC6 staining for polyps of the sessile serrated adenoma type. Further studies, including incorporation of molecular characteristics, are needed to determine if aberrant expression of gastric mucins would prove helpful as an adjunct to morphology in the clinical discrimination of these polyps. Of particular interest, the question of the relationship between MUC6 expression and origin of adenocarcinoma in serrated polyps of the colorectum remains to be answered.

\section{Acknowledgement}

We thank Dr Ray Nagle and staff for carrying out the immunostaining for this project, and Kim-Anh $\mathrm{Vu}$ for color figures. This work was supported by National Cancer Institute grant CA-41108. Immunohistochemical data were generated by the TACMASS Core (Tissue Acquisition and Cellular/ Molecular Analysis Shared Service) that is supported by the Arizona Cancer Center Support Grant, NIH CA023074.

Dr Hamilton is supported by the Frederick F. Becker Distinguished University Chair for Cancer Research.

\section{Disclosure/conflict of interest}

The authors declare no conflict of interest.

\section{References}

1 Longacre TA, Fenoglio-Preiser CM. Mixed hyperplastic adenomatous polyps/serrated adenomas. A distinct form of colorectal neoplasia. Am J Surg Pathol 1990;14: 524-537.

2 Yao T, Kouzuki T, Kajiwara M, et al. 'Serrated' adenoma of the colorectum, with reference to its gastric differentiation and its malignant potential. J Pathol 1999;187:511-517.

3 Matsumoto T, Mizuno M, Shimizu M, et al. Clinicopathological features of serrated adenoma of the colorectum: comparison with traditional adenoma. J Clin Pathol 1999;52:513-516.

4 Hawkins NJ, Gorman P, Tomlinson IP, et al. Colorectal carcinomas arising in the hyperplastic polyposis syndrome progress through the chromosomal instability pathway. Am J Pathol 2000;157:385-392.

5 Sylvester PA, Myerscough $\mathrm{N}$, Warren BF, et al. Differential expression of the chromosome 11 mucin genes in colorectal cancer. J Pathol 2001;195: 327-335.

6 Gurbuz Y, Kloppel G. Differentiation pathways in duodenal and ampullary carcinomas: a comparative study on mucin and trefoil peptide expression, including gastric and colon carcinomas. Virchows Arch 2004;444:536-541.

7 Tanaka M, Kusumi T, Sasaki Y, et al. Colonic intraepithelial carcinoma occurring in a hyperplastic polyp via a serrated adenoma. Pathol Int 2001;51:215-220.

8 Torlakovic E, Skovlund E, Snover DC, et al. Morphologic reappraisal of serrated colorectal polyps. Am J Surg Pathol 2003;27:65-81.

9 Bariol C, Hawkins NJ, Turner JJ, et al. Histopathological and clinical evaluation of serrated adenomas of the colon and rectum. Mod Pathol 2003;16:417-423.

10 Higuchi T, Jass JR. My approach to serrated polyps of the colorectum. J Clin Pathol 2004;57:682-686.

11 Baker K, Zhang Y, Jin C, et al. Proximal versus distal hyperplastic polyps of the colorectum: different lesions or a biological spectrum? J Clin Pathol 2004;57: 1089-1093.

12 Snover DC, Jass JR, Fenoglio-Preiser C, et al. Serrated polyps of the large intestine: a morphologic and molecular review of an evolving concept. Am J Clin Pathol 2005;124:380-391.

13 Sandmeier D, Seelentag W, Bouzourene H. Serrated polyps of the colorectum: is sessile serrated adenoma distinguishable from hyperplastic polyp in a daily practice? Virchows Arch 2007;450:613-618.

14 Torlakovic EE, Gomez JD, Driman DK, et al. Sessile serrated adenoma (SSA) vs traditional serrated adenoma (TSA). Am J Surg Pathol 2008;32:21-29.

$15 \mathrm{Wu}$ JM, Montgomery EA, Iacobuzio-Donahue CA. Frequent beta-catenin nuclear labeling in sessile serrated polyps of the colorectum with neoplastic potential. Am J Clin Pathol 2008;129:416-423.

16 Hawkins NJ, Ward RL. Sporadic colorectal cancers with microsatellite instability and their possible origin in hyperplastic polyps and serrated adenomas. J Natl Cancer Inst 2001;93:1307-1313. 
17 Byrd JC, Bresalier RS. Mucins and mucin binding proteins in colorectal cancer. Cancer Metastasis Rev 2004;23:77-99.

18 Reid CJ, Harris A. Developmental expression of mucin genes in the human gastrointestinal system. Gut 1998;42:220-226.

19 Bartman AE, Sanderson SJ, Ewing SL, et al. Aberrant expression of MUC5AC and MUC6 gastric mucin genes in colorectal polyps. Int J Cancer 1999;80:210-218.

20 Hirono $\mathrm{H}$, Ajioka Y, Watanabe $\mathrm{H}$, et al. Bidirectional gastric differentiation in cellular mucin phenotype (foveolar and pyloric) in serrated adenoma and hyperplastic polyp of the colorectum. Pathol Int 2004;54:401-407.

21 Mochizuka A, Uehara T, Nakamura T, et al. Hyperplastic polyps and sessile serrated 'adenomas' of the colon and rectum display gastric pyloric differentiation. Histochem Cell Biol 2007;128:445-455.
22 Percinel S, Savas B, Ensari A, et al. Mucins in the colorectal neoplastic spectrum with reference to conventional and serrated adenomas. Turk J Gastroenterol 2007;18:230-238.

23 Owens SR, Chiosea SI, Kuan SF. Selective expression of gastric mucin MUC6 in colonic sessile serrated adenoma but not in hyperplastic polyp aids in morphological diagnosis of serrated polyps. Mod Pathol 2008;21:660-669.

24 Alberts DS, Martínez ME, Hess LM, et al. Phase III trial of ursodeoxycholic acid to prevent colorectal adenoma recurrence. J Natl Cancer Inst 2005;97:846-853.

25 Alberts DS, Martínez ME, Roe DJ, et al. Lack of effect of a high-fiber cereal supplement on the recurrence of colorectal adenomas. N Engl J Med 2000;342:1156-1162.

26 Parfitt JR, Driman DK. Survivin and hedgehog protein expression in serrated colorectal polyps: an immunohistochemical study. Hum Path 2007;38:710-717. 